\title{
PRUEBAS DE ESTIMULACION CON GONADOTROPINAS Y CLOMIFEN EN EL ESTUDIO DE LA FUNCION GONADAL
}

\author{
Dr. Elias S. Canales* \\ Dr. Arturo Zárate*
}

El hipotálamo, la hipófisis y los ovarios mantienen una estrecha interelación cíclica que se traduce clínicamente por la menstruación. La secreción de gonadotropinas hipofisiarias está gobernada por neurohormonas proveniente del hipotálamo y éste a su vez, responde a estímulos nerviosos y hormonales. La hormona estimulante del folículo (FSH) y la hormona luteinizante (LH) son secretadas rítmicamente y con cierta relación entre sí para actuar sobre los folículos y desencadenar la ovulación; de esta manera, el folículo roto que liberó al óvulo, se transforma en una nueva glándula de secreción interna: el cuerpo amarillo que produce cantidades importantes de estrógenos y progesterona. Si no ocurre el embarazo, éste cuerpo amarillo involuciona aproximadamente doce días después de su formación y así, al faltar un soporte hormonal, el endometrio se descama (1-4).

Por lo tanto, las alteraciones a cualquier nivel del eje hipotálamo-hipófisis-ovario pueden originar fallas en la ovulación y amenorrea; de ahí la importancia de localizar el sitio afectado dentro de este eje con el fin de realizar el diagnóstico que permi-

ta la selección ed la terapéutica adecuada. La introducción en los últimos diez años de procedimientos inductores de la ovulación, ha permitido entender el mecanismo de este fenómeno y facilitado el diagnóstico de las enfermedades que cursan con amenorrea con lo que se puede establecer el tratamiento eficaz para los procesos anovulatorios.

La presente comunicación tiene por objeto dar a conocer nuestra experiencia con el uso de las gonadotropinas exógenas y el citrato de clomifen, empleados en algunas pruebas dinámicas para el estudio de la función gonadal.

\section{Material y Método}

Para tal fin, se estudió a un grupo constituído por 17 pacientes que consultaron por amenorrea y a las cuales se les administró con fines diagnósticos gonadotropinas o clomifen, de acuerdo a un esquema previamente establecido (Tablas I y II).

* Sección de Ginecoogía Endocrina. Hospital de Gineco Obstetricia Nọ Uno del I.M.S.S. México, D. F. 
TABLA I

\section{PRUEBA DE ESTIMULACION CON GONADOTROPINAS Y CLOMIFEN}

SUERO DE YEGUA EMBARAZADA (PMS)

800 UI, VIA INTRAMUSCULAR, DIARIAS, DURANTE CINCO DIAS.

HORMONA CORIONICA (HGC)

5000 UI, VIA INTRAMUSCULAR, c/ $12 \mathrm{Hr}$, DURANTE CINCO DIAS

GONADOTROPINAS DE LA ORINA DE MUJERES

MENOPAUSICAS (PERGONAL)

$75 \mathrm{UI}$, VIA INTRAMUSCULAR, c/ $12 \mathrm{Hr}$, DURANTE CINCO DIAS

CITRATO DE CLOMIFEN

$100 \mathrm{mg}$, VIA ORAL, DIARIOS, DURANTE CINCO DIAS

En cada una de ellas se efectuó una historia clínica y exploración física detalladas. Con el objeto de valorar la respuesta a estas sustancias, se efectuaron los siguientes estudios previos, durante y después de su administración: registro de la temperatura corporal basal (TCB), moco endocervical, biopsia de endometrio y citología vaginal funcional, además de los siguientes estudios hormonales: cuantificación de gonadotropinas hipofisiarias por medio del bioensayo basado en el crecimiento uterino de ratones prepúberes (5), cuantificación de los 17-hidroxi (6) y 17oxoesteroides (7), estrógenos totales (8), pregnandiol y pregnantriol (9) en orina de 24 horas. En algunos casos se efectuaron además otras pruebas dinámicas para conocer el estado funcional de la suprarrenal (10) así como la reserva hipofisiaria (11). En cinco pacientes se logró obtener, mediante biopsia, material para estudio histológico de la gónada después de las pruebas de estimulación.

\section{Substancias empleadas y Resultados}

Clomifen. El citrato de clomifen es un compuesto no esteroide con estructura química semejante a la del clorotrianiseno y con propiedades biológicas antiestrogénicas. Además, se ha demostrado ampliamente que su administración va seguida de la secreción de gonadotropinas y de estrógenos $(12,13)$. Hasta la actualidad no se ha precisado el mecanismo de acción del clomifén; sin embargo, se sugiere que provoca una secreción de gonadotropinas hipofisiarias, ya sea por un efecto directo sobre el eje hipotálamo-hipófisis y/o disminuyendo la acción inhibitoria que ejercen los estrógenos endógenos sobre los centros neuroendocrinos $(12,13)$. También se ha propuesto una posible acción directa sobre los ovarios que pudiera realizarse modificando la esteroidogénesis o aumentando la sensibilidad folicular a las gonadotropinas endógenos (14). El clomifén solo origina respuesta gonadal en presencia de un eje hipotálamo-hipófisis ovario íntegro, en el cual aparentemente existe una secreción irregular de gonadotropinas ( $F$ $\mathrm{SH} / \mathrm{LH}$ ) que es inadecuada para desencadenar la ovulación. Por lo tanto se puede esperar su acción en presencia de los síndromes clasificados con el común denominador de "estropersistente" (síndrome de ovarios poliquísticos y tecomatosis ovárica) y los de amenorrea galactorera (síndrome de Chiari-Frommel y Del Castillo). Por tal motivo, la inducción de la ovulación en estos casos se puede aprovechar como prueba diagnóstica. 


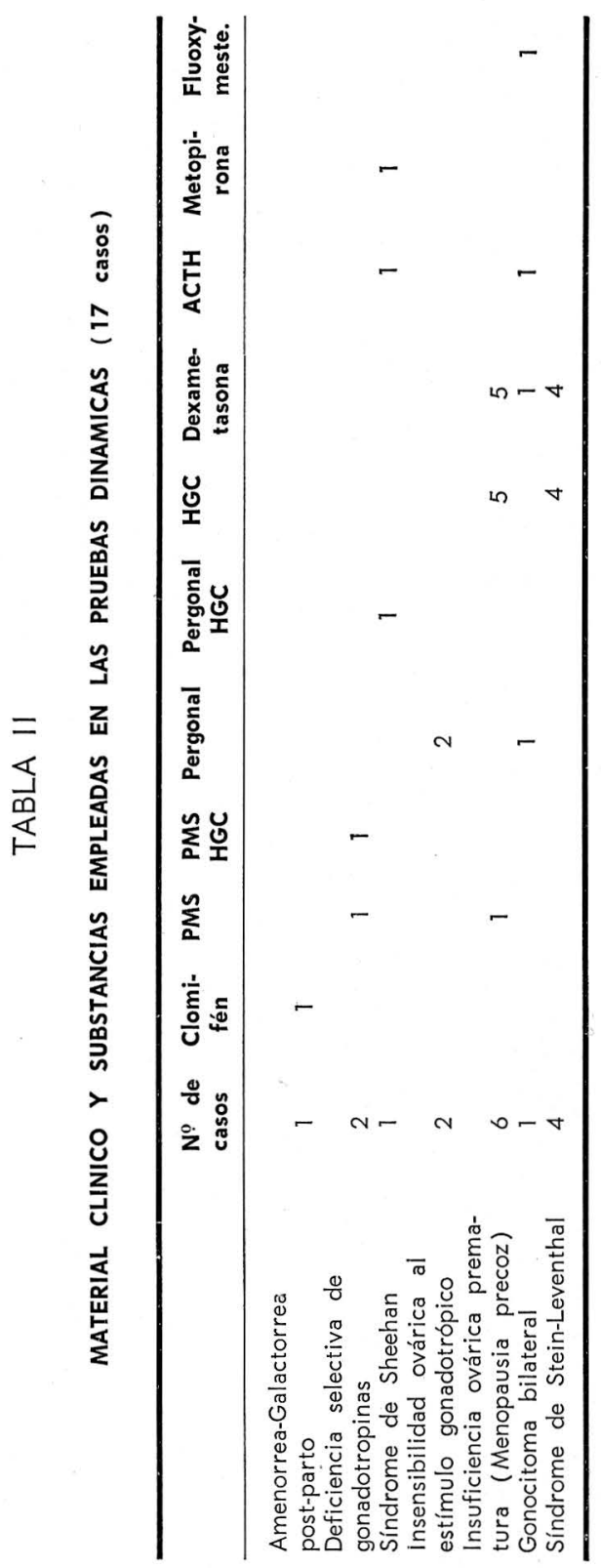

El medicamento (Omifin $\mathrm{R}$ de $\mathrm{Me}$ rrell) se administra por vía oral $y_{\text {, }}$ aunque hay desacuerdo en cuanto a la dosis y duración del estímulo, se puede sugerir como prueba diagnóstica la dosis de $100 \mathrm{mg}$. diarios durante cinco días. Esta prueba se utilizó en una paciente de 33 años de edad con amenorrea y galactorrea post parto, obteniéndose una buena respuesta al medicamento. La cantidad de gonadotropinas hipofisiarias excretadas en la orina aumentó, y simultáneamente se pudo conseguir esteroidogénesis ovárica y ovulación.

(Fig. № 1 ).

Gonadotropinas exógenas. El uso de estas hórmonas permite establecer el diagnóstico diferencial entre la amenorrea por lesión intrínseca irreversible de los ovarios y la que aparece por falta del estímulo gonadotrópico. Este hecho es de gran importancia porque determina con gran seguridad en futuro en cuanto a la fertilidad de la paciente, con lo que se evitan procedimientos diagnósticos prolongados y costosos. La estimulación con gonadotropinas puede hacerse ya sea con sustancias que tienen actividad biológica de tipo FSH o con actividad LH.

Sustancias con actividad FSH. Durante mucho tiempo se utilizó una sutancia extraída del suero de la yegua embarazada, hormona gonadotrópica sérica (PMS), como estimulante de la función gonadal; sin embargo, el uso repetido de esta hormona favoreció en desarrollo de anticuerpos y por tal motivo se emplea con poca frecuencia en la actualidad con fines terapéuticos (15). En tres pacientes se realizó una prueba de estimulación con PMS (Gestyl R de Organón, Holanda). La primera de ellas fue una enferma de 36 años de edad, con amenorrea secundaria, esterilidad, "bochornos", hipoestrogenismo y 
PRUEBA DE ESTIMULACION CON CLOMIFEN

(SINDROME DE AMENORREA-GALACTORREA POST-PARTO)

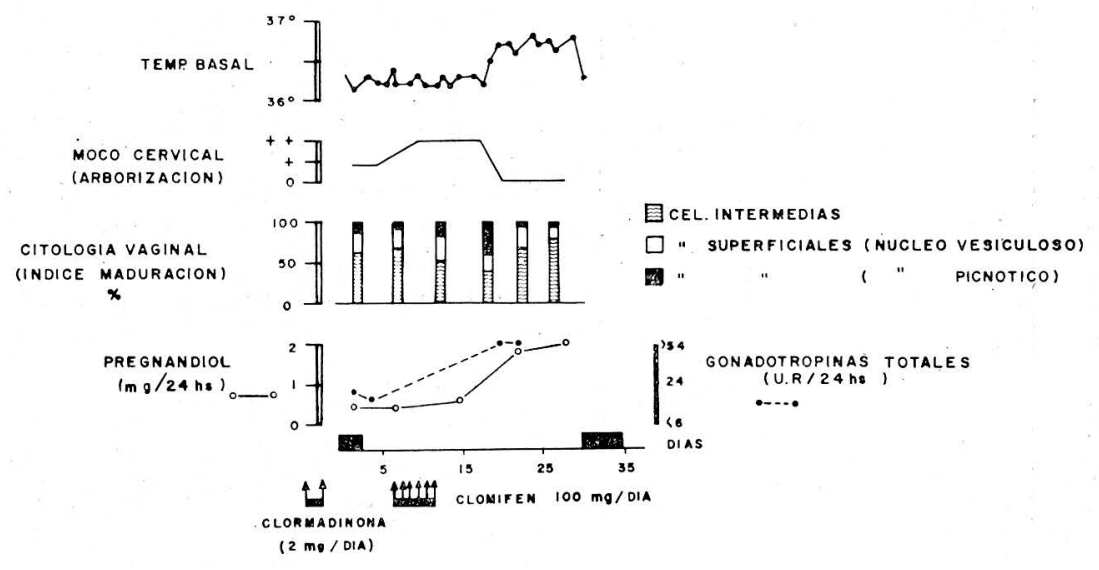

FIGURA Nọ 1

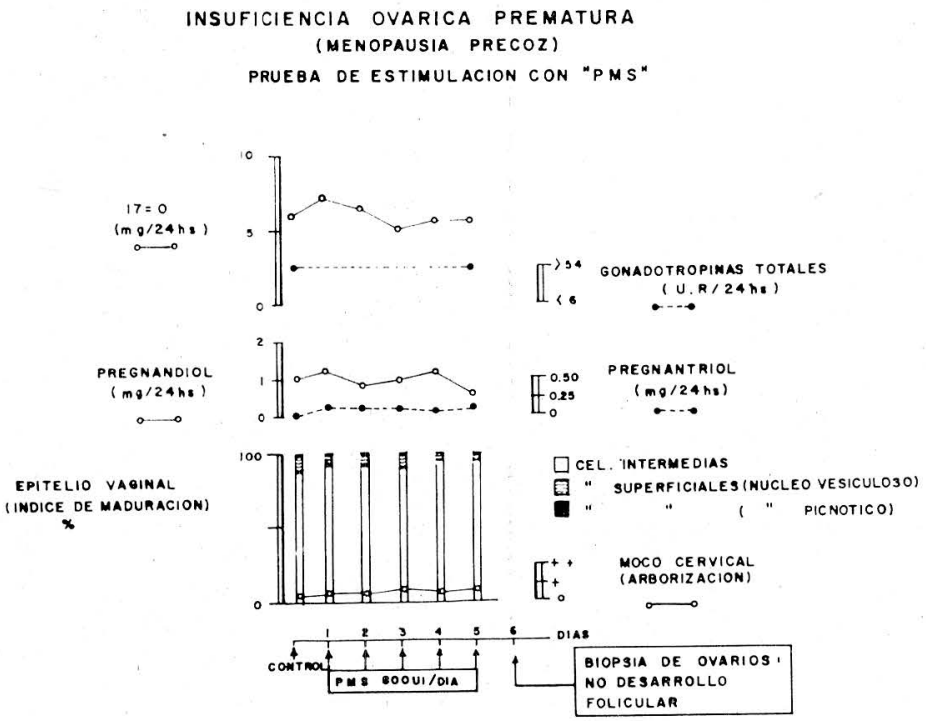

FIGURA $N^{0} 2$ 
elevación de las gonadotropinas totales urinarias, en la cual se sospechó el diagnóstico de insuficiencia ovárica prematura (menopausia precoz). La administración de PMS no logró inducir esteroidogénesis ovárica y al estudio histológico del ovario, no se observó crecimiento folicular. (Fig. No 2). El segundo caso se trató de una paciente de 29 años de edad con amenorrea primaria y deficiencia selectiva de gonadotropinas que, con la mostraron evidente actividad hormonal demostrada por un aumento en la producción de estrógenos aunque no se logró la ovulación. Para de- mostrar la efectividad del PMS se repitió la prueba de estimulación con Pergonal, observándose los mismos resultados. El estudio histsológico del ovario demostró la presencia de desarrollo folicular y luteinización de la teca interna. (Fig. № 3). Finalmente, el empleo de PMS asociado a la gonadotropina coriónica (HGC) en una paciente de 31 años de edad con diagnóstico de deficiencia selectiva de gonadotropinas, en la cual el estudio radiológico de la silla turca demostró la existencia de un tumor hipofisiario, también fue capaz de estimular al ovario produciendo un au-

DEFICIENCIA SELECTIVA DE GONADOTROPINAS

PRUEBA DE ESTIMULACION

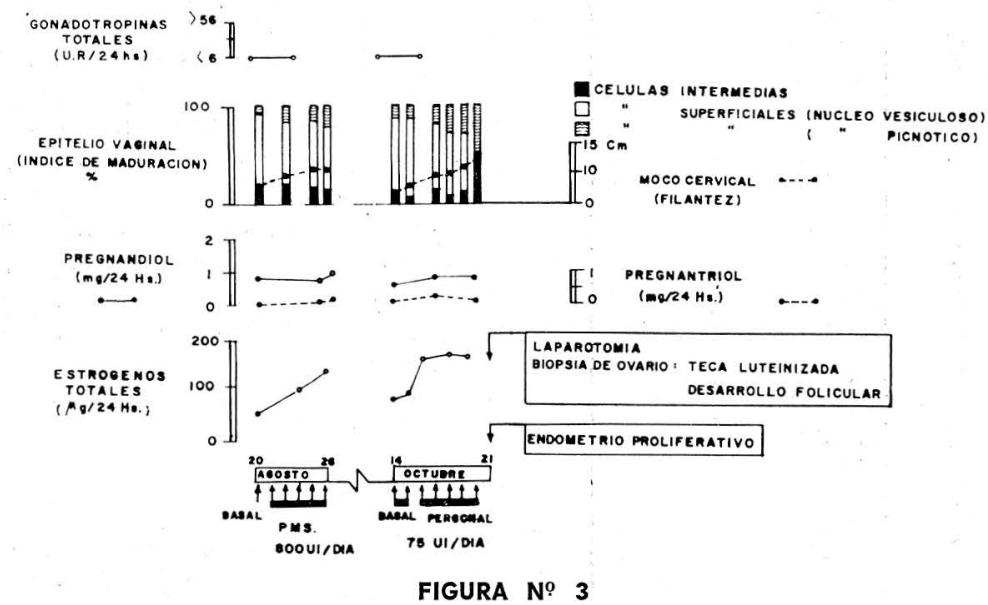

TABLA III

PRUEBA DE ESTIMULACION OVARICA EN UNA PACIENTE CON DEFICIENCIA SELECTIVA DE GONADOTROPINAS POR TUMOR HIPOFISIARIO

\begin{tabular}{|c|c|c|c|c|c|c|}
\hline & $\frac{E T}{(u g / 24 h r .)}$ & $\begin{array}{c}\text { EXCRECION } \\
17-\mathrm{OH}\end{array}$ & $\begin{array}{l}\text { URINARIA } \\
17-0 \\
\mathrm{mg} / 24\end{array}$ & $\begin{array}{l}\text { Pdiol } \\
\text { hr. }\end{array}$ & Ptriol & $\begin{array}{l}\text { Citología Vag. } \\
\text { (Indice de } \\
\text { maduración) }\end{array}$ \\
\hline $\begin{array}{l}\text { Basal } \\
4{ }^{\circ} \text { día PMS ( } 800 \text { UI/día) más }\end{array}$ & 8.0 & 5.6 & 11.7 & 0.4 & 0.2 & $78 / 22 / 0$ \\
\hline HGC $(10,000$ UI/día $)$ & 32.0 & 5.4 & 14.0 & 0.3 & 0.6 & $20 / 75 / 5$ \\
\hline
\end{tabular}




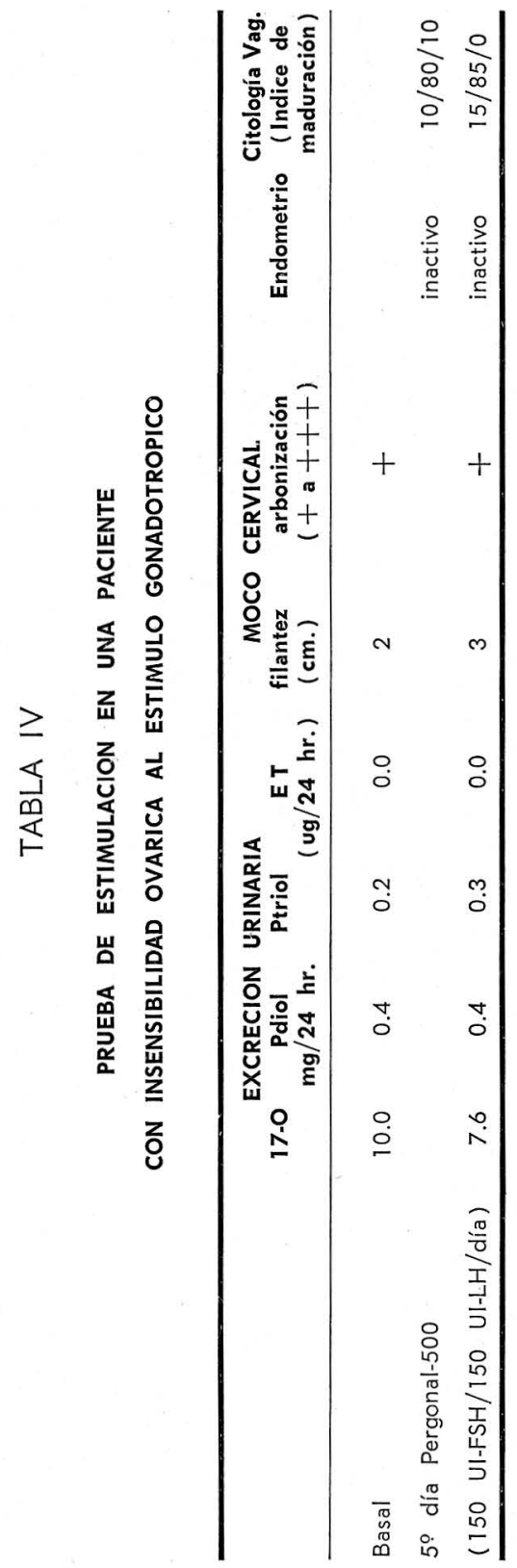

mento en la excreción de estrógenos aunque tampoco se logró producir ovulación ya que la excreción de pregnandiol y pregnantriol no se modificó. (Tabla № III).

En 1958 se logró obtener FSH de extractos hipofisiarios de seres humanos (16) y posteriormente de la orina de mujeres post menopáusicas (17). Por la facilidad de obtener esta última hormona, se le utiliza más frecuentemente en las pruebas de estimulación gonadal; esta sustancia se presenta para su uso en forma liofilizada con una actividad de $75 \mathrm{UI}$ de FSH más 75 UI de LH de acuerdo al 2: IRP (Second International Reference Preparation) (OMS, 1964). La prueba utilizada por nosotros consistió en la administración de 150 UI de Pergonal-500 (Laboratorios Cutter) (Lote 199/193), diariamente por vía intramuscular, durante cinco días. Esta sustancia fue empleada en cinco casos; en la paciente con deficiencia selectiva de gonadotropinas que había respondido previamente a la aplicación de PMS, también lo hizo con la admniistración de Pergonal (Fig. № 3 ).

En dos pacientes con diagnóstico de hipogonadismo por insensibilidad ovárica al estímulo gonadotrópico, ambas de 26 años de edad, amenorrea primaria, esterilidad e hipoestrogenismo, cuyo estudio completo ha sido informado previamente (18), la administración de Pergonal demostró la ausencia de respuesta a la estimulación, ya que ni los niveles hormonales ni los índices biológicos se modificaron. Al estudio histológico, los ovarios mostraron la presencia de abundantes folículos primordiales sin evidencia de desarrollo (Fig. N ? 4 y Tabla № IV). 


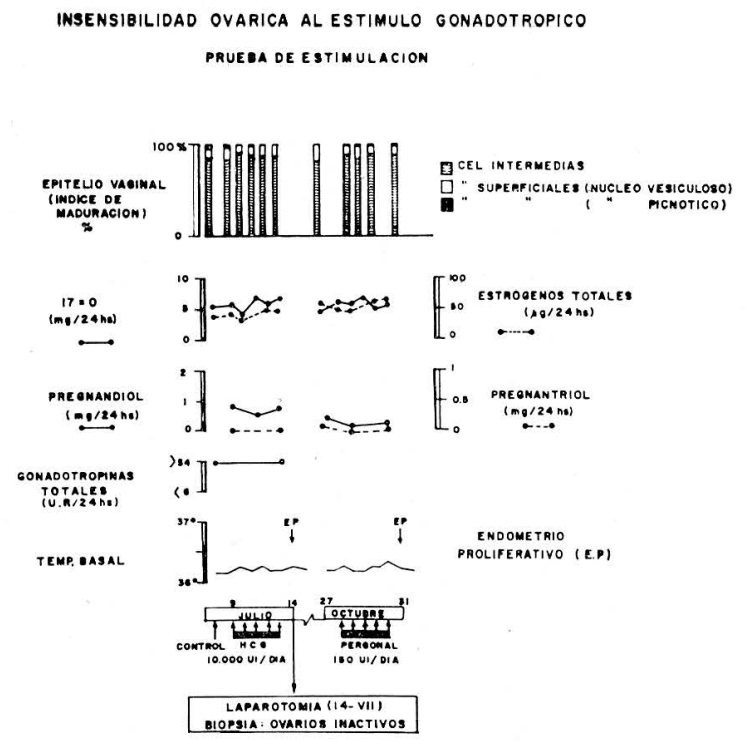

FIGURA № 4

Por otra parte, en una paciente de 30 años de edad con síndrome de Sheehan de cuatro años de evolución, se efectuó, además de las pruebas de estimulación suprarrenal con ACTH y de reserva hipofisiaria con Metopirona, una prueba de estimulación gonadal con Pergonal asociado a gonadotropina coriónica (HCG), con lo cual se pudo obtener una adecuada respuesta ovárica evidenciada por un aumento en la excreción de estrógenos urinarios así como demostrar su efecto biológico a nivel del epitelio vaginal. (Tabla $\mathrm{N}$ : $\mathrm{V}$ ).

Finalmente, la administración de Pergonal en una paciente de 16 años de edad, con un gonocitoma ovárico bilateral que cursó con amenorrea primaria y clitoromegalia sin signos de virilización, se observó un aumento en la excreción urinaria de estrógenos; las pruebas de estimulación e inhibición suprarrenal fueron normales y la administración de Fluoxy- mesterona fue incapaz de inhibir la producción de las gonadotropinas hipofisiarias (Tabla № VI).

Sustancias con actividad LH. Generalmnete se utiliza la gonadotropina coriónica (HGC) que tiene actividad biológica semejante a la LH. La prueba con HGC tiene valor menor en cuanto a su efectividad gonadotrópica; sin embargo, se le puede utilizar cuando no se cuente con sustancias con actividad FSH. Por otra parte, las gonadotropinas también han resultado útiles en el diagnóstico diferencial de la amenorrea que cursa con hirsutismo (19) y que puede ser de naturaleza ovárica o suprarrenal. La prueba consiste básicamente en suprimir la función suprarrenal con dexametasona para así determinar la producción androgénica de esta glándula y posteriormente, mientras se mantiene la inhibición, se hace la estimulación ovárica ya sea con FSH o con LH, lo cual permite a su vez re- 
TABLA V

PRUEBAS DE RESERVA HIPOFISIARIA, ESTIMULACION SUPRARRENAL

Y ESTIMULACION GONADAL, EN UNA PACIENTE CON SINDROME DE SHEEHAN

\begin{tabular}{|c|c|c|c|c|c|c|c|}
\hline 1 & $\begin{array}{c}\text { GHT } \\
(\mathrm{ur} / 24 \mathrm{hr} .)\end{array}$ & $\begin{array}{l}\text { EXCRECION } \\
\text { ET } \\
(\mathrm{vg} / 24 \mathrm{hr} .)\end{array}$ & $\begin{array}{l}\text { URINARIA } \\
\text { 17-OH }\end{array}$ & $\begin{array}{l}17-0 \\
\mathrm{mg} \cdot / 24\end{array}$ & $\begin{array}{l}\text { Pdiol } \\
\text { hr. }\end{array}$ & Ptriol & $\begin{array}{c}\text { Citología Vag. } \\
\text { (Indice de } \\
\text { maduración) }\end{array}$ \\
\hline $\begin{array}{l}\text { Basal } \\
5^{0} \text { día Pergonal-500 }\end{array}$ & -6 & 11.3 & 3.0 & 4.8 & 0.45 & 0.30 & $0 / 100 / 0$ \\
\hline $\begin{array}{l}\text { ( } 150 \text { UI-FSH/150 UI-LH/día) } \\
5^{\circ} \text { día Gonadotropina Coriónica }\end{array}$ & 一 & 32.0 & 2.0 & 4.8 & 0.45 & 0.41 & $0 / 90 / 10$ \\
\hline$(10,000$ UI/día) más Pergonal-500 & - & 44.0 & 2.0 & 5.0 & 1.03 & 0.51 & $0 / 88 / 12$ \\
\hline Basal & & & 3.9 & 3.2 & & & \\
\hline Metopirona ( $3 \mathrm{~g} /$ día/oral) & & & 3.9 & 3.2 & & & \\
\hline $\begin{array}{l}\text { Basal } \\
\text { ACTH }(25 \text { UI en } 500 \mathrm{ml} \text { de sol. salina }\end{array}$ & & & 2.2 & 2.5 & & & \\
\hline durante ocho horas) & & & 7.2 & 12.5 & & & \\
\hline
\end{tabular}

TABLA VI

PRUEBAS DE ESTIMULACION E INHIBICION SUPRARRENAL Y GONADAL EN UNA PACIENTE CON GONOCITOMA BILATERAL

\begin{tabular}{|c|c|c|c|c|c|c|c|}
\hline & $\begin{array}{c}\text { GHT } \\
\text { (ur/24 hr.) }\end{array}$ & $\begin{array}{l}\text { EXCRECION } \\
\text { E T } \\
(\mathrm{ug} / 24 \mathrm{hr} .)\end{array}$ & $\begin{array}{l}\text { URINARIA } \\
\text { I7-OH }\end{array}$ & $\begin{array}{l}17-0 \\
\mathrm{mg} \cdot / 24\end{array}$ & $\begin{array}{l}\text { Pdiol } \\
\text { hr. }\end{array}$ & Ptriol & $\begin{array}{l}\text { Citología Vag. } \\
\text { (Indice de } \\
\text { maduración) }\end{array}$ \\
\hline $\begin{array}{l}\text { Basal } \\
\text { ACTH ( } 25 \text { Ul en } 500 \mathrm{ml} \text {. de sol. }\end{array}$ & +54 & 2.3 & 10.7 & 4.5 & 0.3 & 0.1 & $99 / 1 / 0$ \\
\hline $\begin{array}{l}\text { salina durante ocho horas) } \\
4^{\circ} \text { día de Dexametasona }\end{array}$ & - & 3.0 & 14.0 & 16.0 & 0.4 & 0.7 & $100 / 0 / 0$ \\
\hline 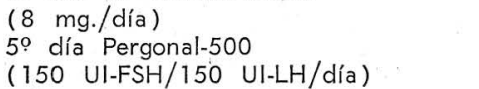 & 一 & 2.6 & 4.0 & 0.2 & 0.2 & 0.1 & $98 / 2 / 0$ \\
\hline $\begin{array}{l}\text { más Dexametasona } \\
5^{0} \text { día de Fluoxymesterona }\end{array}$ & - & 23.6 & 15.0 & 1.0 & 0.6 & 0.8 & $90 / 10 / 0$ \\
\hline $\begin{array}{l}\text { (60 mg./día) } \\
\text { Una semana después }\end{array}$ & +54 & 23.7 & 8.3 & 6.2 & 0.5 & 0.7 & $92 / 8 / 0$ \\
\hline $\begin{array}{l}\text { de gonadectomía } \\
\text { Seis meses después de gonadectomía }\end{array}$ & +54 & 0.0 & 7.0 & 8.0 & 0.2 & 0.2 & $99 / 1 / 0$ \\
\hline (estrogenoterapia) & 12 & - & 6.5 & 3.0 & 0.2 & 0.0 & \\
\hline
\end{tabular}


conocer aproximadamente la participación del ovario en la síntesis de andrógenos. Aunque la prueba se puede valorar con los cambios en la excreción de los 17-oxoesteroides totales, se obtienen resultados más precisos cuando se realiza la estimación fraccionada de estos corticoides y/o de la cantidad de testosterona en plasma o en orina (20).

Para tal fin, se empleó una prueba de estimulación con gonadotropina coriónica (HGC) (APL R Ayerst) a la dosis de 5.000 UI cada doce horas por vía intramuscular, durante cinco días, previa inhibición suprarre- nal, en dos grupos de pacientes. El primero de ellos constituído por cuatro enfermas con síndrome de SteinLeventhal e hirsutismo (Tabla № VII). Se pudo observar que la excreción de los 17-oxoesteroides basales disminuyeron con la administración de la dexametasona y posteriormente estos se elevaron después de la administración de la gonadotropina coriónica. El segundo grupo, formado por cinco pacientes con diagnóstico clínico de menopausia precoz, en las cuales se siguió el mismo procedimiento anotado previamente, no se observó respuesta ovárica a pesar de la adecuada inhibición suprarrenal (Tabla № VIII).

TABLA VII

PRUEBAS DE INHIBICION SUPRARRENAL Y ESTIMULACION OVARICA EN CUATRO PACIENTES CON SINDROME DE STEIN-LEVENTHAL

\begin{tabular}{|c|c|c|c|c|c|c|}
\hline \multirow{2}{*}{\multicolumn{3}{|c|}{ Días de estudio }} & \multicolumn{4}{|c|}{$\begin{array}{c}\text { Excreción urinaria de } 17-0 x \text { xoesteroides } \\
\mathrm{mg} . / 24 \mathrm{hr}\end{array}$} \\
\hline & & & 1 & 2 & 3 & 4 \\
\hline $1-2$ & Basal & & 16.4 & 15.8 & 14.3 & 12.0 \\
\hline $3-6$ & $\begin{array}{l}4^{9} \text { día de Dexametasona } \\
\text { (8 } \mathrm{mg} / \text { / día) }\end{array}$ & & 4.8 & 3.6 & 8.0 & 4.2 \\
\hline $7-11$ & $\begin{array}{c}\text { 59 día de Gonadotropina } \\
(10,000 \mathrm{UI} / \mathrm{día}) \\
\text { más Dexametasona }\end{array}$ & Coriónica & 10.9 & 9.2 & 15.0 & 12.3 \\
\hline
\end{tabular}

TABLA VIII

PRUEBA DE ESTIMULACION OVARICA EN CINCO PACIENTES CON MENOPAUSIA PRECOZ

\begin{tabular}{|c|c|c|c|c|c|c|c|}
\hline \multirow{2}{*}{\multicolumn{2}{|c|}{ Días de estudio }} & & \multicolumn{5}{|c|}{$\begin{array}{c}\text { Excreción urinaria de } 17-0 x \text { oesteroides } \\
\mathrm{mg} . / 24 \mathrm{hr} \text {. }\end{array}$} \\
\hline & & & 1 & 2 & 3 & 4 & 5 \\
\hline $1-2$ & Basal & & 5.2 & 7.0 & 6.0 & 13.3 & 11.7 \\
\hline $3-6$ & $\begin{array}{l}\text { 49 día de Dexametasona } \\
\text { ( } 8 \mathrm{mg} \text {. díal }\end{array}$ & & 3.2 & 3.2 & 4.0 & 5.3 & 4.3 \\
\hline $7-11$ & $\begin{array}{c}5^{0} \text { día de Gonadotropina } \\
\text { ( } 10,000 \text { UI/día }) \\
\text { más Dexametasona }\end{array}$ & Coriónica & 2.9 & 3.6 & 3.6 & 4.8 & 4.3 \\
\hline
\end{tabular}




\section{Conclusiones}

Por todo lo anterior, se puede concluir que las pruebas dinámicas son auxiliares valiosos en el estudio de la función gonadal y permiten por lo tanto, una mayor precisión en el diagnóstico de los problemas que afectan al eje hipotálamo-hipófisisovario. El uso de las gonadotropinas facilita el diagnóstico diferencial entre al amenorrea por lesión intrínseca del ovario y la causada por algún problema hipotálamo-hipofisiario. Así, la falta de respuesta al estímulo gonadotrópico se observa en la disgenesia gonadal, en la castración quirúrgica y en la menopausia precoz. En cambio, cuando el problema se localiza en la esfera secretora de gonadotropinas, como ocurre en la deficiencia selectiva de las mismas y en el síndrome de Sheehan, el ovario es capaz de responder al estímulo exógeno. Así mismo, la prueba de estimulación ovárica combinada con la supresión suprarrenal es de utilidad en los casos de amenorrea e hirsutismo. Finalmente, el uso del citrato de clomifén ayuda a establecer el diagnóstico en los síndromes de estro persistente y en la amenorrea denominada "hipotalámica" o "psicógena".

\section{Resumen}

Se revisan los factores endocrinos que determinan la menstruación y se concluye que el uso de sustancias capaces de inducir ovulación como las gonadotropinas exógenas y el citrato de clomifén, son de utilidad en el diagnóstico diferencial de algunos casos de amenorrea, permitiendo establecer si el factor causal es primariamente ovárico o secundario a un padecimiento hipotálamo hipofisiario. Se hace mención de las dosis empleadas en la prueba de estimulación y se presentan los resultados obtenidos en un grupo de pacientes estudiadas; asímismo, se establece el criterio para interpretar la respuesta.

\section{Summary}

A revision of endocrine factors that determine menstruation is made. The use of substances capable of inducing ovulation such as exogenic gonadotropins and Clomifen Citrate are useful for differential diagnosis of some cases of amenorrhea, allowing for the establishment of whether the causative factor is primarily ovarian or secondarily hypothalamichypophyseal.

Mention is made of the doses used in the stimulatory test and the obtained results are shown in the group of patients studied; the criteria are established to interpret the answer.

\section{BIBLIOGRAFIA}

1 WALLACH, E. E.: Endocrinology of ovulation. Clin. Obstet. Gynec. 10: 361, 1967.

2 STEVENS, V. C.: Comparison of FSH and LH patterns in plasma, urine and urinary extracts during the menstrual cycle. J. Clin. Endocr. 29: 94, 1969.

3 STROTT, C. A., YOSHIMI, T., ROSS, G. T. and LIPSETT, M. B.: Ovarian physiology: Relationship between plasma $\mathrm{LH}$ and steroidogenesis by the follicle and corpus lutem; effect of HGC. J. Clin. Endocr. 29: 1157, 1969.

4 GREENBLATT, R. B., MAHESH, V. B., ZARATE, A.: La ovulación en el humano. Rev. Invest. Clin. 18: 477, 1966.

5 KLINEFELTER, H. F., ALBRIGHT, F., and GRISWOLD, G. C.: Experience with a quantitative test for normal or decreased amounts of follicle stimulating hormone in the urine in endocrinological diagnosis. J. Clin. Endocr. 3: 529, 1943.

6 PORTER, C. C., and SILBER, R. H.: A quantitative color reaction for cortisone and related 17, 21-dihydroxi - 20 ketosteroids. J. Biol. Chem. 185: 201, 1950.

7 DREKTER, I. J., PEARSON, S., BARTESAK, E., and MCGAVACK, T. H.: A rapid method for the determination of total urinary 17 ketosteroids. J. Clin. Endocr. 7: 795, 1947. 
8 BELING, C. G.: Gel fitration of conjugated urinary estrogens and its applications in clinical assay. Acta Endocr. 43 (supp, 79): 9, 1963.

9 GOLDZIEHER, J. W., and NAKAMURA, Y.: A clinical method for the determination of urinary pregnandiol and pregnantriol. Acta Endocr. 41: 371, 1962.

10 JENKINS, D., FORSHAM, P. H., LAIDLAW, J. C., REDDY, W. J. and THORN, G. W. : Use of ACTH in the diagnosis of adrenal cortical insufficiency. Amer. J. Med. 18: 3, 1955.

11 LIDDLE, G. W., ESTEP, H. L., KENDALL, J. W., WILLIAMS, W. C. and TOWNES, A. W.: Clinical application of a new test of pituitary reserve. J. Clin. Endocr. 19: 875, 1959.

12 GREENBLATT, R. B., ZARATE, A., MAHESH, V. B.: Inducción e inhibición de la ovulación. Editado por C. Gual. Excerpta Medica Foundation. Amsterdam, 1966, pág. 59.

13 VORYS, N., GANTT, C. L., HAMWI, G. J., COPELAND, W. E., and ULLERY, J. C.: Clinical utility of chemical induction of ovulation. Amer. J. Obstet. Gynec. 88: 425, 1964.

14 SMITH, O. W., SMITH, G. V., and KISTNER, R. W.: Action of MER-25 and of clomiphe- ne on the human ovary. J.A.M.A. 184: $878,1963$.

15 GREENBLATT, R. B., ZARATE, A., MAHESH, B.: Methods availables for the induction of ovulation. Proc. Sixth. Pan-Amer. Congr. Endocr. Excerpta Medica Foundation. Int. Congr. Series num. 112. Amsterdam, 1966. Pág. 129.

16 GEMZELL, C. A., DICZFALUSY, E., and TILINGER, K. G.: Clinical effect of human pituitary FSH. J. Clin. Endocr. 18: 1333, 1958.

17 LUNNENFELD, B., MENSI, A. and VOLET, B.: Clinical effect of human post-menopausal gonadotrophin. Proc. First International Congress of Endocrinoogy. Periodica. Copenhagen, 1960, pág. 587.

18 ZARATE, A., VALENZUELA, S., CANALES, E. S.: Hipogonadismo ocasionado por insensibilidad ovárica al estímulo gonadotrópico. Ginec. Obstet. Mex. 28: 409, 1970.

19 MA.HESH, V. B., and GREENBLATT, R. B.: Urinary steroid excretion patterns in hirsutism. II. Effect of ovarian stimulation with human pituitary FSH on urinary 17-Ketosteroids. J. Clin. Endocr. 24: 1293, 1964.

20 ISMAIL, A. A. A. and LORAINE, J. A. : A disorder frequently associated with menstrual abnormalities. Clin. Obstet. Gynec. 12: $800,1969$. 